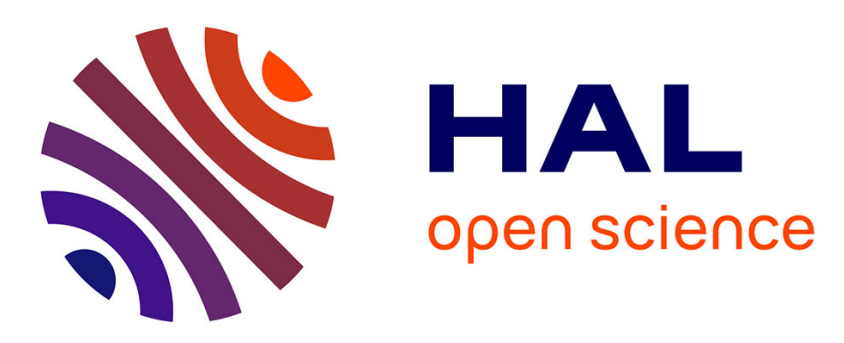

\title{
A New Optimized Denoising Method applied to the Spot World Heritage Initiative and its Spot 5 Supermode images
}

Antoine Masse, Christophe Latry, Julien Nosavan, Simon Baillarin, Sébastien Lefèvre

\section{To cite this version:}

Antoine Masse, Christophe Latry, Julien Nosavan, Simon Baillarin, Sébastien Lefèvre. A New Optimized Denoising Method applied to the Spot World Heritage Initiative and its Spot 5 Supermode images. IGARSS 2018 - 2018 IEEE International Geoscience and Remote Sensing Symposium, 2018, Valencia, Spain. 10.1109/IGARSS.2018.8518357 . hal-01969010

\section{HAL Id: hal-01969010 https://hal.science/hal-01969010}

Submitted on 13 Nov 2019

HAL is a multi-disciplinary open access archive for the deposit and dissemination of scientific research documents, whether they are published or not. The documents may come from teaching and research institutions in France or abroad, or from public or private research centers.
L'archive ouverte pluridisciplinaire HAL, est destinée au dépôt et à la diffusion de documents scientifiques de niveau recherche, publiés ou non, émanant des établissements d'enseignement et de recherche français ou étrangers, des laboratoires publics ou privés. 


\section{A New Optimized Denoising Method applied to the Spot World Heritage Initiative and its Spot 5 Supermode images}

\author{
Antoine Masse, Christophe Latry, Julien Nosavan, Simon Baillarin \\ French Spatial Agency (Centre National d'Etudes Spatiales) \\ 18, avenue Edouard Belin, 31401 Toulouse cedex 9, France \\ Email: firstname.lastname@cnes.fr
}

\author{
Sébastien Lefèvre \\ Univ. Bretagne-Sud \\ UMR 6074, IRISA 56000 Vannes, France \\ Email: firstname.lastname@ irisa.fr
}

\begin{abstract}
In the context of the Spot World Heritage (SWH) initiative, the long term archive of Spot 1 to 5 satellite data will be reprocessed. This initiative gives the opportunity to increase Spot data quality and production efficiency with the introduction of new state-of-the-art processing methods. In this context, we consider the Spot 5 Supermode processing chain and the introduction of a new denoising method. Our objective is to propose the best denoising method in terms of efficiency and accuracy for the Spot 5 Supermode data production. We report two experimentations that lead to the choice of the Non Local Bayes (NLB) denoising method; it is fast, accurate and remains stable in terms of efficiency and quality for different landscapes and image sizes. We also introduce two optimized versions of the NLB algorithm that increase the computation efficiency by a factor up to 4 .
\end{abstract}

Index Terms-Spot World Heritage, Spot, Supermode, Restoration, Denoising, Non Local Bayes

\section{INTRODUCTION}

Spot World Heritage (SWH) is an initiative of the French Spatial Agency (CNES) and aims to preserve and promote Spot 1 to 5 archives by providing new enhanced products to users. Over the last 30 years, Spot 1 to 5 satellites have collected more than 25 million images over the world [1]. The SWH initiative will use Big Data technologies for processing, storage and management of the huge volume of data. First products are expected in 2018, and all the Spot 1 to 5 collection will be available before 2020. These new Spot products will provide a past extension for Sentinel-2 time series and will increase application opportunities.

This initiative has two main objectives. First, the long-term archive of Spot 1 to 5 satellite data will be reprocessed and provided to users at Level $1 \mathrm{~A}$, i.e. basic radiometric correction and preliminary cloud cover estimation. The second objective is to enhance L1A products to match with ESA Sentinel-2 standards with the introduction of two new processing levels for Spot data: L1B (geometric corrections with use of Sentinel2 Global Reference Images and Digital Elevation Model, and enhanced radiometric corrections) and L1C (ortho-rectification and tiling according to the Sentinel-2 global reference). If these methods and processing chains are well known and validated, the SWH initiative is also an opportunity to increase data quality and production efficiency with the introduction of new state-of-the-art processing methods.

In this context, we consider the Spot 5 Supermode processing chain [2] which is used to create a super-resolution image (2.5 meter resolution) from two simultaneous Spot 5 panchromatic acquisitions (5 meter resolution). A quincunx resampling is applied to combine these two images to obtain a 2.5-meter image. This resampling produces blur and noisy images due to high spatial frequencies, thus a linear deconvolution is applied for deblurring. With the current Spot 5 Supermode product, noise is still present but, hopefully, this noise is well known and has a signal dependent variance. A simple variance stabilization operation [3] can be used to transform this noise model to a zero-mean Gaussian noise i.e. white noise. In the early 2000s, during the development of the Spot 5 ground segment, there was no effective and accurate enough denoising method which could be deployed in a data production system. Since then, new denoising methods have emerged to remove efficiently and accurately white-noise in optical satellite images.

From our previous work on denoising Pléiades satellite imagery [4] and an exhaustive denoising method taxonomy [5] [6], we have identified five state-of-the-art denoising methods: Non Local Means (NLM) [7], Non Local Bayes (NLB) [8], Bayes Least Squares Gaussian Scale Mixture (BLS-GSM) [9], Block-Matching and 3D filtering (BM3D) [10], and KSingular Value Decomposition (K-SVD) [11]. In addition to these methods, we also present an optimized version of the Non Local Bayes method used for Pléiades VHR images [12] with the introduction of new techniques to reduce statistical overestimations and thus to increase efficiency.

Our objective is to experiment and choose the best denoising method in terms of efficiency and accuracy with applications to the Spot 5 Supermode data production.

\section{STATE-OF-THE-ART DENOISING METHODS APPLIED TO SPOT 5 SUPERMODE}

In this section, we present two experiments to choose the best denoising method in terms of efficiency and accuracy for Spot 5 Supermode products. 
(a)
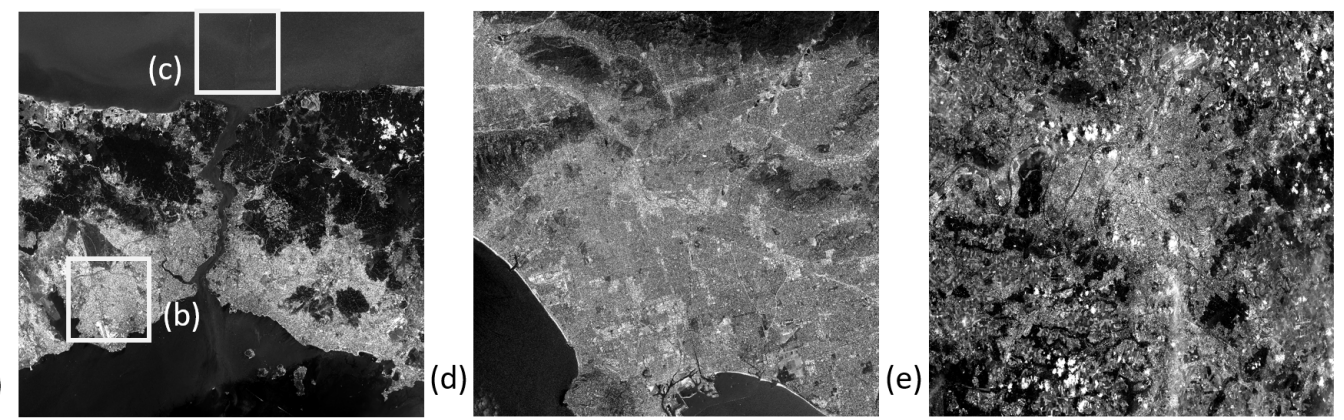

Fig. 1. Illustrations of the Spot 5 Supermode products used in the experimentations: (a) Istanbul, Turkey (24K by $24 \mathrm{~K}$ pixels), (b) Istanbul city (4K by $4 \mathrm{~K}$ ), (c) Black sea (4K by $4 \mathrm{~K})$, (d) Los Angeles, USA (24K by $24 \mathrm{~K})$, and (e) Paris, France (24K by $24 \mathrm{~K})$.

(a)
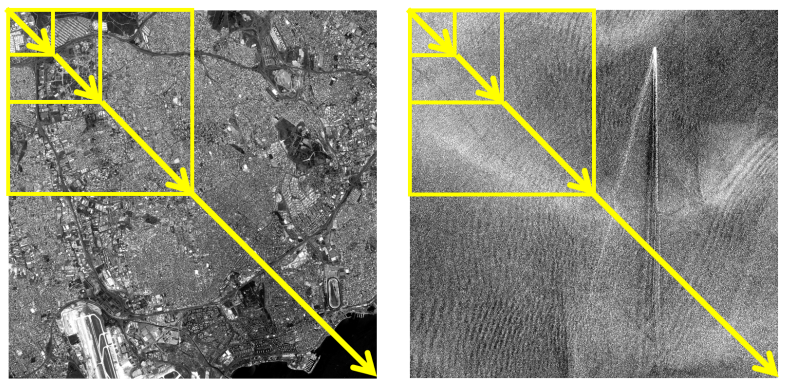

(b)

Fig. 2. Extracted datasets (4096 by 4096 pixels) from Istanbul full Spot 5 Supermode image: (a) Istanbul town and (b) Black Sea. For scalability experimentation, smaller images are extracted as illustrated by the yellow squares and arrows, from size 32 by 32 to 4096 by 4096 pixels.

\section{A. Experimentation context}

Five datasets have been used for the experimentations: three full Spot 5 Supermode datasets over Istanbul (Turkey), Los Angeles (USA), and Paris (France) and two smaller representative datasets extracted from the Istanbul full one (see Fig. 1). These two small datasets contain specific landscapes. The first one, called "Istanbul town", is centered over the city of Istanbul and mainly contains urban areas. The second one, called "Black sea", is centered over the sea surface and mostly contains wave textures (see Fig. 2).

1) Method implementations: Implementations and optimal parameterizations have been extracted from the IPOL database (www.ipol.im) and are noted NLB [13], NLM [14], BLSGSM [15], BM3D [16] and K-SVD [17].

2) Architecture: For the experimentations, we have used a High Performance Computing (HPC) platform based on Lenovo NX360m5 with Intel Xeon E5-2650 v4 (2.2-2.9GHz) processors, 128 GB RAM, CentOS 7.2 / Gdal 2.1.1 / Fftw 3.3.4 / GCC 4.8.2, and managed with PBS pro v13. We have restricted this architecture to a single-core execution in order to evaluate the efficiency per core.

3) Evaluation measures: We evaluate the results with two quantitative measures: the well-known Peak Signal-to-Noise Ratio (PSNR) and the number of pixels denoised per second. The PSNR is used as a distortion measure that quantifies reconstruction quality between a denoised image and a reference one. The number of pixels denoised per second is used to measure efficiency and only reflects the performance of the algorithm core, without any IO nor non-denoising operations.

4) Experiment protocol: As a noise-free reference is unknown in real data production, we use a specific experiment protocol. First, we consider a denoised image as reference which has been selected as the best result by a panel of photo-interpret experts. This reference image is then corrupted through artificial introduction of white noise $\left(\sigma^{2}=1\right)$. Each experiment is repeated ten times to avoid negative influence from the random noise simulation. Finally, we evaluate each result and summarize them by computing mean and standard deviation of both the PSNR and the number of pixels denoised per second.

\section{B. Experiment 1: comparison of state-of-the-art denoising methods}

For the first experimentation, we consider images with different sizes extracted from the two small datasets as illustrated in Fig. 2. Starting from the upper left corner, image size grows from 32 by 32 pixels to 4096 by 4096 pixels. This growing experimentation is used to evaluate the consistency of denoising method results over various image sizes. PSNR and number of pixels denoised per second results are presented in Fig. 3.

As far as PSNR is concerned and for the "Istanbul town" dataset (see Fig. 3 (a)), NLB and K-SVD are the best methods followed by BM3D. BLS-GSM fails to produce correct results for large images. NLM is the worst and is not accurate as it has similar PSNR values than the noisy image. For the "Black Sea" dataset (see Fig. 3 (b)), K-SVD, NLB and BM3D are the best methods. NLM is far behind and BLS-GSM produces the best results for small image sizes but then PSNR value drastically decreases when image size increases.

If we compare the number of pixels denoised per second, we can observe that for the "Istanbul town" dataset (see Fig. 3 (c)), NLB and NLM are the quickest; BLS-GSM and BM3D are about 2 times slower and K-SVD is 5 times slower. For the "Black sea" dataset (see Fig. 3 (d)), results are quite similar except for K-SVD, which is slower than NLB and NLM but closer. BLS-GSM and BM3D are the slowest. The difference for K-SVD results between the two datasets is explained by the nature of the datasets and the method category. K-SVD is a 

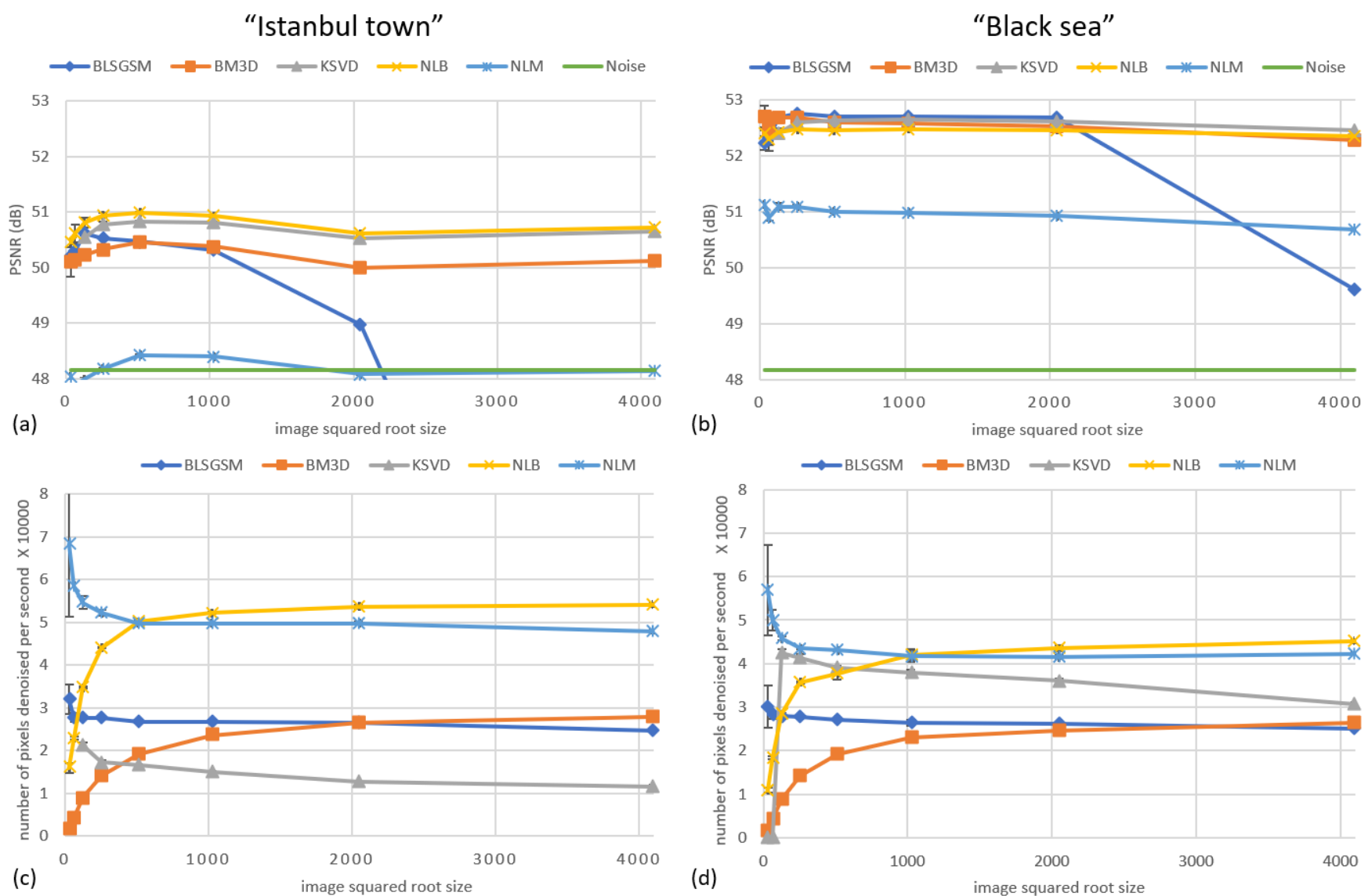

Fig. 3. Comparison of the state-of-the-art denoising results for the two datasets "Istanbul town" (a, c) and "Black sea" (b, d). Experimentation is made using variable image size from 32 by 32 to 4096 by 4096 pixels. Each result is expressed as the mean and the standard deviation for 10 white-noise $\left(\sigma^{2}=1\right)$ simulations of the PSNR (a, b) and the number of pixels denoised per second (c, d).

dictionary based method; which means that the more complex the image content is, the longer is the training.

In conclusion of the state-of-the-art denoising method comparison for both datasets, we have observed that NLB and $\mathrm{K}-\mathrm{SVD}$ are the best methods in terms of PSNR. We have also observed that NLB and NLM are the most efficient. Thus, the Non-Local Bayes method appears to be the best compromise; it is fast, accurate and remains stable in terms of efficiency and quality for different landscapes and image sizes. Please also note that for full scene size ( $24 \mathrm{~K}$ by $24 \mathrm{~K}$ pixels), BM3D, KSVD and BLS-GSM implementations fail to produce a result, even with the use of image splitting techniques.

\section{Experiment 2: efficiency optimization for the Non Local Bayes denoising method}

In the second experimentation, we compare the original NLB method with an optimized version that we previously introduced and experimented on Pléiades images [12]. This optimization consists in the addition of a new parameter used to reduce the number of statistical estimations made by the NLB algorithm. In the following, we present two parameterizations of this new optimized NLB. The first version, called "NLB Opt", reduces by half the number of estimations while the second one, called "NLB Opt Fast", reduces this number to its minimum while preserving statistical significance (requirement for the application of the law of large numbers).

In the Tab. I, we present the denoising results for the two small datasets for a fixed size of 4096 by 4096 pixels. We can observe that "NLB Opt" PSNR results are very close to the classic NLB but this new version is about 1.5 time quicker. The second version, "NLB Opt Fast", is less accurate than the classic NLB but still equivalent or better in terms of PSNR than other state-of-the-art denoising methods. However, the speed gain by using "NLB Opt Fast" is important compared to other methods. For example, "NLB Opt Fast" is 4 times quicker than classic NLB for the three full images.

Conclusions are similar when considering full datasets $(24 \mathrm{~K}$ by $24 \mathrm{~K}$ pixels). In Tab. II, we can observe that "NLB Opt" and "NLB Opt Fast" are respectively 1.5 and 4 times quicker than classic NLB.

\section{CONCLUSION AND PERSPECTIVES}

As part of the SWH initiative, the number of Spot 5 Supermode products to be denoised is up to $415 \mathrm{~K}$. In this context, the Non Local Bayes (NLB) denoising method is the best choice as it is efficient and accurate. We also experiment two optimized versions of the NLB algorithm, called "NLB Opt" and "NLB Opt Fast", which increases the efficiency by 
TABLE I

COMPARISON OF FIVE REPRESENTATIVE STATE-OF-THE-ART DENOISING METHODS AND TWO OPTIMIZED VERSIONS OF THE NLB METHOD FOR TWO SMALL DATASETS (4096 BY 4096 PIXELS). EACH EXPERIMENT IS EXPRESSED AS THE MEAN AND THE STANDARD DEVIATION OF THE DENOISING RESULTS FOR 10 WHITE-NOISE $\left(\sigma^{2}=1\right)$ SIMULATIONS.

\begin{tabular}{l|rr|rr}
\hline & \multicolumn{2}{|c}{ Istanbul town } & \multicolumn{2}{c}{ Black Sea } \\
\hline Method & PSNR(dB) & Pixels.s-1 & PSNR(dB) & Pixels.s-1 \\
\hline BLS-GSM & $37.11 \pm 0.00$ & $24733 \pm 131$ & $49.62 \pm 0.00$ & $25084 \pm 182$ \\
BM3D & $50.11 \pm 0.00$ & $27817 \pm 112$ & $52.29 \pm 0.00$ & $26524 \pm 52$ \\
K-SVD & $50.65 \pm 0.00$ & $11640 \pm 254$ & $52.45 \pm 0.01$ & $30766 \pm 334$ \\
NLB & $50.72 \pm 0.00$ & $53932 \pm 296$ & $52.35 \pm 0.00$ & $45924 \pm 146$ \\
NLM & $48.14 \pm 0.00$ & $47918 \pm 429$ & $50.68 \pm 0.00$ & $42302 \pm 251$ \\
\hline NLB opt & $50.70 \pm 0.00$ & $77325 \pm 217$ & $52.32 \pm 0.00$ & $71422 \pm 240$ \\
NLB opt fast & $50.62 \pm 0.00$ & $214597 \pm 1327$ & $52.15 \pm 0.00$ & $235867 \pm 2658$ \\
\hline
\end{tabular}

TABLE II

COMPARISON OF RESULTS FOR NLB AND ITS OPTIMIZED VERSIONS "NLB OPT" AND "NLB OPT FAST" FOR THE 3 FULL DATASETS (24K BY 24K PIXELS). EACH EXPERIMENT IS EXPRESSED AS THE MEAN AND THE STANDARD DEVIATION OF THE DENOISING RESULTS FOR 10 WHITE-NOISE $\left(\sigma^{2}=1\right)$ SIMULATIONS.

\begin{tabular}{l|rrr|rrr|rrr}
\hline & \multicolumn{3}{|c}{ Istanbul } & \multicolumn{3}{c}{ Los Angeles } & \multicolumn{2}{c}{ Paris } \\
\hline Method & PSNR(dB) & Pixels.s-1 & Accel. & PSNR(dB) & Pixels.s-1 & Accel. & PSNR(dB) & Pixels.s-1 & Accel. \\
\hline NLB base & $52.37 \pm 0.00$ & $48858 \pm 86$ & & $51.45 \pm 0.00$ & $53547 \pm 75$ & & $53.50 \pm 0.00$ & $51100 \pm 67$ \\
NLB Opt & $52.36 \pm 0.00$ & $75986 \pm 148$ & 1.56 & $51.43 \pm 0.00$ & $77942 \pm 188$ & 1.46 & $53.44 \pm 0.00$ & $64051 \pm 105$ & 1.25 \\
NLB Opt fast & $52.22 \pm 0.00$ & $224309 \pm 368$ & 4.59 & $51.34 \pm 0.00$ & $217097 \pm 334$ & 4.05 & $53.25 \pm 0.00$ & $227375 \pm 157$ & 4.45 \\
\hline
\end{tabular}

a factor up to 4 . With a production system of $2 \mathrm{~K}$ processing cores, it will take 27 days for all Spot 5 Supermode product denoising with classic NLB. This processing time could be reduced to respectively 18 and 6 days with the two optimized versions, respectively "NLB Opt" and "NLB Opt Fast". As a research perspective, we will also evaluate the denoising impact on further applications like Digital Terrain Model (DTM) generation or Land Cover Mapping.

\section{ACKNOWLEDGMENT}

We would like to thank the French Spatial Agency (CNES) for Spot images providing and computation facilities. This work is partially founded by CNES postdoctoral grant.

\section{REFERENCES}

[1] J. Nosavan, A. Moreau, A. Masse, B. Chausserie-Lapre, and C. Caillet, "Spot World Heritage: Spot 1-5 data curation and valorization with new enhanced SWH products," in Proc. of the 2017 conference on Big Data from Space (BiDS17), Toulouse, France, Nov. 2017, pp. 225-228.

[2] B. R. Christophe Latry, "In-flight commissioning of SPOT5 THR quincunx sampling mode," in Proc. SPIE 4881, Sensors, Systems, and Next-Generation Satellites VI, vol. 4881, Crete, Greece, 2003, pp. 4881 - 4892. [Online]. Available: http://dx.doi.org/10.1117/12.462632

[3] F. J. Anscombe, "The transformation of Poisson, binomial and negative-binomial data," Biometrika, vol. 35, no. 3/4, pp. 246-254, 1948. [Online]. Available: http://www.jstor.org/stable/2332343

[4] A. Masse, S. Lefevre, R. Binet, S. Artigues, P. Lassalle, G. Blanchet, and S. Baillarin, "Fast and accurate denoising method applied to very high resolution optical remote sensing images," in Proc. SPIE. 10427, Image and Signal Processing for Remote Sensing XXIII, vol. 10427, Varsaw, Poland, 2017, p. 20. [Online]. Available: http://dx.doi.org/10.1117/12.2277705

[5] L. Shao, R. Yan, X. Li, and Y. Liu, "From Heuristic Optimization to Dictionary Learning: A Review and Comprehensive Comparison of Image Denoising Algorithms," IEEE Transactions on Cybernetics, vol. 44, no. 7, pp. 1001-1013, Jul. 2014
[6] V. Katkovnik, A. Foi, K. Egiazarian, and J. Astola, "From Local Kernel to Nonlocal Multiple-Model Image Denoising," International Journal of Computer Vision, vol. 86, no. 1, p. 1, 2009. [Online]. Available: http://dx.doi.org/10.1007/s11263-009-0272-7

[7] A. Buades, B. Coll, and J. M. Morel, "A Review of Image Denoising Algorithms, with a New One," Multiscale Modeling \& Simulation, vol. 4, no. 2, pp. 490-530, 2005.

[8] M. Lebrun, A. Buades, and J. M. Morel, "A Nonlocal Bayesian Image Denoising Algorithm," SIAM Journal on Imaging Sciences, vol. 6, no. 3, pp. 1665-1688, 2013. [Online]. Available: http://dx.doi.org/10.1137/120874989

[9] J. Portilla, V. Strela, M. J. Wainwright, and E. P. Simoncelli, "Image denoising using scale mixtures of Gaussians in the wavelet domain," IEEE Transactions on Image Processing, vol. 12, no. 11, pp. 13381351, Nov. 2003

[10] K. Dabov, A. Foi, V. Katkovnik, and K. Egiazarian, "Image Denoising by Sparse 3-D Transform-Domain Collaborative Filtering," IEEE Transactions on Image Processing, vol. 16, no. 8, pp. 2080-2095, Aug. 2007.

[11] M. Elad and M. Aharon, "Image Denoising Via Sparse and Redundant Representations Over Learned Dictionaries," IEEE Transactions on Image Processing, vol. 15, no. 12, pp. 3736-3745, Dec. 2006.

[12] A. Masse, S. Lefvre, R. Binet, S. Artigues, G. Blanchet, and S. Baillarin, "Denoising very high resolution optical remote sensing images: Application and optimization of nonlocal bayes method," IEEE Journal of Selected Topics in Applied Earth Observations and Remote Sensing, vol. 11, no. 3, pp. 691-700, March 2018.

[13] M. Lebrun, A. Buades, and J.-M. Morel, "Implementation of the "Non-Local Bayes" (NL-Bayes) Image Denoising Algorithm," Image Processing On Line, vol. 3, pp. 1-42, 2013.

[14] A. Buades, B. Coll, and J.-M. Morel, "Non-Local Means Denoising," Image Processing On Line, vol. 1, pp. 208-212, 2011.

[15] B. Rajaei, "An Analysis and Improvement of the BLS-GSM Denoising Method," Image Processing On Line, vol. 4, pp. 44-70, 2014.

[16] M. Lebrun, "An Analysis and Implementation of the BM3d Image Denoising Method," Image Processing On Line, vol. 2, pp. 175-213, 2012.

[17] M. Lebrun and A. Leclaire, "An Implementation and Detailed Analysis of the K-SVD Image Denoising Algorithm," Image Processing On Line, vol. 2, pp. 96-133, 2012. 